Article

\title{
Continuous Reusability using Immobilized HasApf in Chemoenzymatic Deracemization: A New Heterogeneous Enzyme Catalysis
}

\author{
Hiroyuki Nagaoka \\ Sanyo Shokuhin Co., Ltd. R \& D, 555-4 Asakura, Maebashi, Gunma 371-0811, Japan; \\ hnagaoka@sanyofoods.co.jp; Tel.: +81-27-220-3471 \\ Academic Editor: Emily Weinert \\ Received: 5 August 2016; Accepted: 14 October 2016; Published: 25 October 2016
}

\begin{abstract}
This study found that the calibration curve of heme acquisition system A (Has A, a new reactive active species) immobilized by a porous ceramic particle (ImHApf; immobilized HasA from Pseudomonas fluorescens) can be constructed in the range of $1750-1450 \mathrm{~cm}^{-1}$ using Fourier transform infrared spectroscopy (FTIR) analysis, and evaluated its catalytic efficiency. In the asymmetric oxidation of rac-1-(6-methoxynaphthalen-2-yl)ethanol (rac-1: a naproxen precursor), a product ketone from the $(R)$-isomer is desymmetrized using $\mathrm{NaBH}_{4}$ and continuously reused even if treated with an organic solvent in $50 \mathrm{mM}$ glycine- $\mathrm{NaOH}$ buffer at $40{ }^{\circ} \mathrm{C}$ in the absence of nicotinamide adenine dinucleotide (NAD(P)), leading to $>99 \%$ enantiomeric excess and $>90 \%$ chemical yield; the activity was calculated at $0.74 \pm 0.03 \mathrm{mU} /(\mathrm{mg} \cdot \mathrm{min})$ and the turnover number was determined to be approximately $2 \times 10^{6}$. It was confirmed that the other sec-alcohols such as

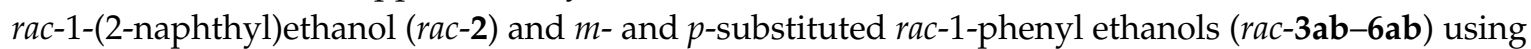
ImHApf can also yield a single stereoisomer from a racemate. Therefore, HasA immobilization can be expected to become an important tool for building an environmentally friendly system that promotes industrial sustainability.
\end{abstract}

Keywords: immobilized HasA; iron electron-transfer system; porous ceramic particle; continuous reusability; chemoenzymatic deracemization

\section{Introduction}

The asymmetric oxidation of secondary alcohols (sec-alcohols) using a heme-binding protein incorporating an iron electron-transfer system as an enzymatic catalyst has been established in organic synthesis [1], whereas the mechanism of heme uptake by heme acquisition system A (HasA), a heme-binding protein which is secreted by various gram-negative pathogens, has been reported [2-4]. The advantages of immobilized or heterogeneous biocatalysts over those that are "free" in solution are well known and include stability, reusability, convenience in continuous operation, and volumetric productivity [5]; there is increasing interest in the use of tougher polymeric materials, particularly inorganic ceramic supports. For example, inorganic carriers for enzyme support represent an important topic in organic synthesis, and porous ceramic particles prepared from kaolin minerals are well suited for the enzymatic production of various biochemicals, such as pharmaceuticals, agricultural chemicals, and their precursors [6]. If the asymmetric oxidation system of HasA could be immobilized by a porous ceramic particle (ImHApf; immobilized HasA from Pseudomonas fluorescens), this new system would not only be able to overcome comparison to dehydrogenase enzymatic systems using coenzyme nicotinamide adenine dinucleotide $(\mathrm{NAD}(\mathrm{P}))$, but could also be used to evaluate the efficiency and turnover of HasA in ImHApf using Fourier transform infrared spectroscopy (FTIR) analysis [7]. 
Over the past decade, the use of biomaterials for asymmetric oxidation has been examined [8], i.e., the redox activity of the pea protein immobilized by $\mathrm{Ca}^{2+}$-alginate gel (IPP; immobilized pea protein) is available for the kinetic resolution of sec-alcohols [9], as shown in Scheme 1, e.g., rac-1-(6-methoxynaphthalen-2-yl)ethanol (rac-1: a naproxen precursor), rac-1-(2-naphthyl)ethanol (rac-2), rac-1-octen-3-ol, and $m$ - and $p$-substituted rac-1-phenyl ethanols (rac-3ab-6ab) [10]. Specifically, membrane-bound enzymes (MEs) eluted from IPP after aeration can be activated by a glycine- $\mathrm{NaOH}$ buffer ( $\mathrm{pH}$ 9.0-10.0) in the absence of $\mathrm{NAD}(\mathrm{P})$ [11], and they can be applied to turnover kinetic resolutions, synthesizing (S)-(+)-1 via a polyethylene glycol-coated ME [12]. The key reaction for asymmetric oxidation lies in an iron electron transfer system based on an oxygen-driven catalytic system [13], and the nature of the species showed 93\% similarity with a $20.853 \mathrm{kDa}$ hemophore HasA expressed by Escherichia coli BL21(DE3) (HasApf) after using an N-terminal sequence comparison [14]. Therefore, it is suggested that the successive asymmetric catalytic events can be regenerated using an incorporated iron electron transfer system [15], which is similar to that utilized by the oxygen-driven cytochrome P450 [16]. There are some structural differences between cytochrome P450 and HasA: namely, the key structural features of cytochrome P450 are that heme iron is coordinated by a cysteine ligand in the proximal site [17] and is open for oxygen binding in the distal site [18], resulting in a better system to activate oxygen and catalyze oxidations, whereas the set of HasA ligands appears to create an unlikely system because the heme iron is already coordinated [19], that is, a histidine is the ligand in the proximal site, and a tyrosine is coordinated by the distal site [20]. Therefore, it has been previously demonstrated that HasA functions at the distal site, so that oxygen will bind for oxygen activation to be oxoferric ( $\mathrm{Fe}(\mathrm{III})-\mathrm{O}-\mathrm{O}^{-}$) species and so that it will coordinate solely in the promotion of oxoferryl $(\mathrm{Fe}(\mathrm{IV})=\mathrm{O})$ species. Moreover, one study showed that the further explorations of HasA should be regarding efficiency, turnover number, or enzyme loading in comparison with other known systems for this transformation or similar transformations [21].

The construction of deracemization methods that obtain a single enantiomer from a sec-alcohol [22] is classified according to the stereochemical course of enzymatic and chemical reactions [23], and the one-pot deracemization of biochemicals, such as pharmaceuticals, agricultural chemicals, and their precursors, is emphasized [24]. Two notable issues are whether ImHApf can be evaluated using a FTIR calibration curve and whether cyclic deracemization with continuous reusability can occur via ImHApf [25]. Therefore, this study aimed to (1) construct a FTIR calibration curve using different concentrations of HasA in ImHApf; (2) evaluate the turnover number and activity; (3) clarify the cyclic deracemization process using ImHApf and $\mathrm{NaBH}_{4}$; and (4) present ImHApf as a new asymmetric oxidation catalysis tool.

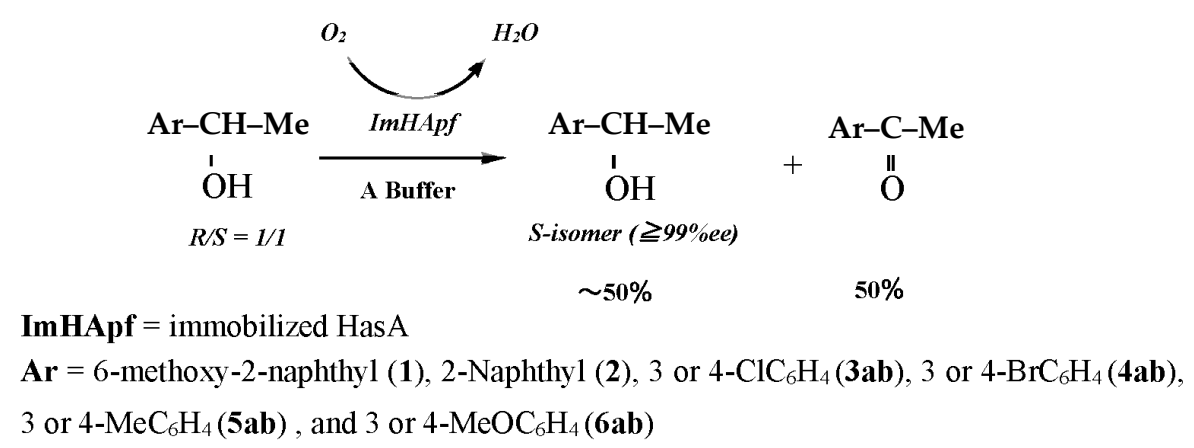

Scheme 1. Kinetic resolution of $r a c-\mathbf{1}$ or $r a c-2$ or $r a c-3 a b-6 a b$ using immobilized HasA from Pseudomonas fluorescens (ImHApf). ee: enantiomeric excess. 


\section{Results}

\subsection{Application to the Immobilization of HasApf}

\subsubsection{Immobilization of HasApf with a Toyonite}

The Toyonite family, consisting of porous ceramic particles prepared from kaolin minerals, exhibits differences in adsorption onto hydrophobic or hydrophilic surfaces because of the modification of four types of organic functional groups. As shown in Figure 1, the Toyonites $(1.0 \mathrm{~g})$ were suspended in a HasApf solution $(18 \mathrm{mg} / 10 \mathrm{~mL})$ containing a $50 \mathrm{mM}$ potassium phosphate buffer $(\mathrm{pH} 9.0)$ for $50 \mathrm{~h}$, and ImHApf was synthesized in the four Toyonites. The four Toyonites ( $2 \mathrm{mg}$ each) were reacted with rac-1 (0.8 mM:0.4 mg) in a $50 \mathrm{mM}$ glycine-NaOH buffer $(4.0 \mathrm{~mL})$ for $50 \mathrm{~h}$. The results indicate that Toyonite 200 was most suitable for HasA immobilization/adsorption because of a resulting lower HasA leach (referring to the degree of HasA (\%) that remains in the filtrate after filtering the suspension) and a higher product ketone and optical purity; thus, HasA can be effectively immobilized/adsorbed onto Toyonite, which features an organic hydroxyl group (in the case of Toyonite 200), a methacryloyloxy (Toyonite 200M), phenylamino (Toyonite 200P), or amino group (Toyonite 200A) [5]. This result means that HasA may be a hydrophilic moiety in the structure and that Toyonite 200 plays an important role in the immobilization. In addition, it was speculated that, compared with other immobilization techniques (e.g., utilizing a $1.0 \%(\mathrm{v} / \mathrm{v})$ aqueous $\mathrm{CaCl}_{2}$ solution for $\mathrm{Ca}^{2+}$-alginate gel beads [9] or a $0.5 \%(\mathrm{v} / \mathrm{v})$ glutaraldehyde solution for cross-linking [10]), HasA can be simply and effectively immobilized into porous ceramic particles (particularly, Toyonite 200) without additional chemicals.

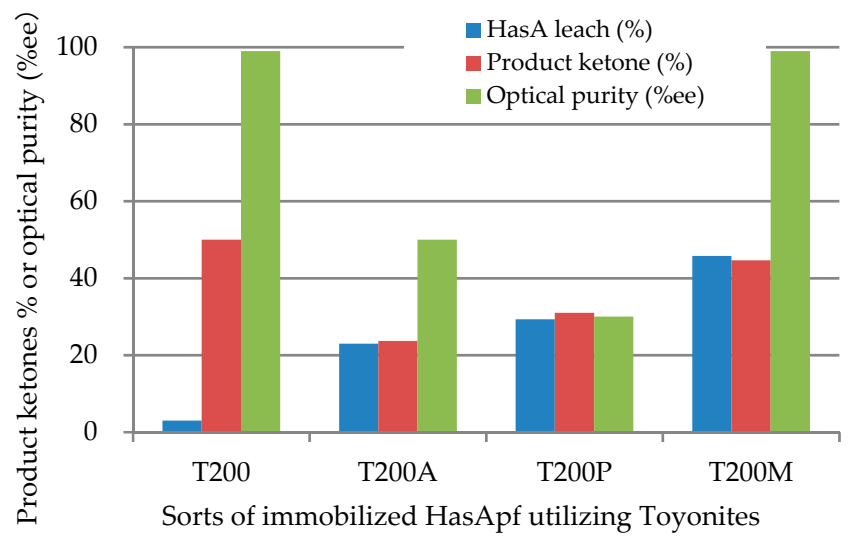

Figure 1. Asymmetric oxidation activity using immobilized heme acquisition system A expressed by Escherichia coli BL21(DE3) (ImHApf) and rac-1 (0.8 mM) in a $50 \mathrm{mM}$ glycine-NaOH buffer (4.0 mL); HasA leach (blue: 100\% maximum) and product ketone (red: 50\% maximum) were monitored, suggesting that the degree of heme acquisition system A (HasA) leaching from ImHApf may be in the lowest level of Toyonites (i.e., T200 < T200A < T200P < T200M). ee: enantiomeric excess.

\subsubsection{FTIR Analysis for the Detection of HasA Spectra in ImHApf}

For the first time, a calibration curve of HasA was constructed using FTIR, a portable attenuated total reflection (ATR) instrument (A2 Technologies, ML version: S.T. Japan Inc., Tokyo, Japan) and the significance of spectra in HasApf/ImHApf were studied in detail. As shown in Figure 2, dried HasApf, immobilized HasApf (i.e., ImHApf), and Toyonite 200 were monitored via FTIR spectroscopy. Although the FTIR spectra of immobilized HasApf are similar to those of Toyonite 200, significant differences were detected in the range of $1750-1450 \mathrm{~cm}^{-1}$. This finding may be applicable to both the calibration of HasA in ImHApf and quality control of the ImHApf product. 


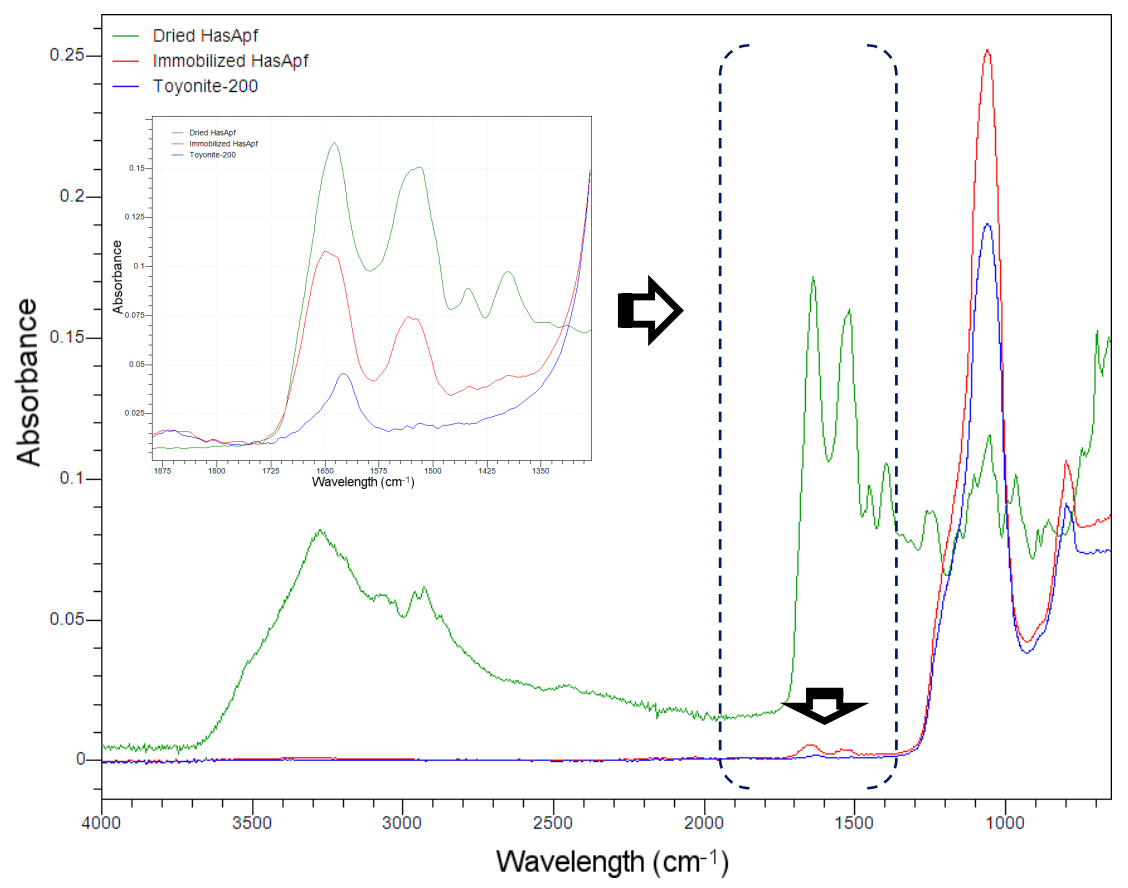

Figure 2. Different Fourier transform infrared (FTIR) spectra of dried heme acquisition system A expressed by Escherichia coli BL21(DE3) (HasApf), immobilized HasApf, or Toyonite 200. HasA spectra in ImHApf were located in the range of $1750-1450 \mathrm{~cm}^{-1}$.

\subsubsection{Construction of the HasA Calibration Curve in ImHApf}

To further generate a calibration curve for the quality control of the product, ImHApf was prepared with different concentrations of HasA, and immobilization was performed using Toyonite 200 (1 g) per HasApf/potassium phosphate solution (2.25, 4.5, 9, and $18 \mathrm{mg} / 10 \mathrm{~mL})$. As shown in Figure 3a, the concentration of HasApf in ImHApf was successfully monitored in the range of $1750-1450 \mathrm{~cm}^{-1}$. In addition, as shown in Figure $3 \mathrm{~b}$, the calibration curve was successfully constructed for the immobilization to 16-18 mg HasA [5], with predicted mg of HasA in the $y$ axis and actual mg of HasA in the $x$ axis. Thus, FTIR analysis is an ideal method for the quality control of the product because HasA leach can be experimentally evaluated after filtering the suspension (Figure 1). The advantages of immobilized ImHApf over the "free" ones in solution include stability, reusability, and convenience in continuous operation and are further examined below.

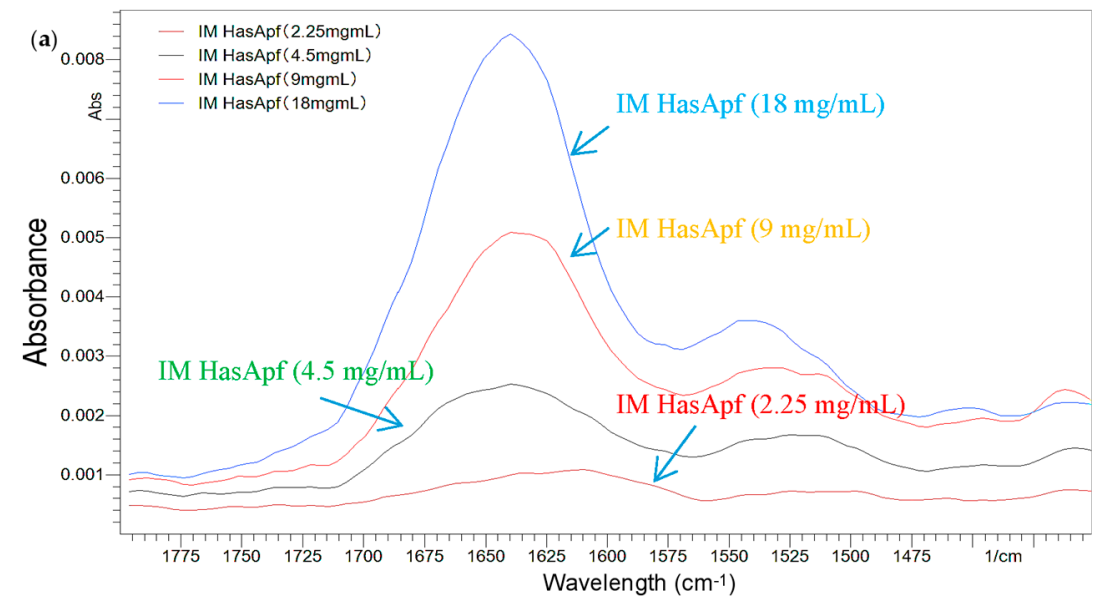

Figure 3. Cont. 


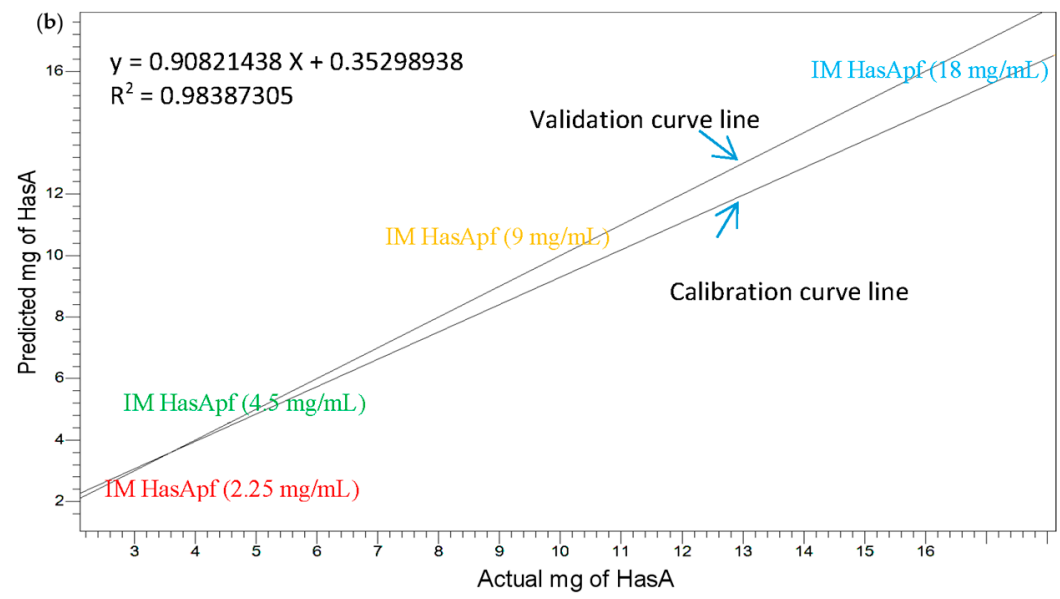

Figure 3. Construction of the HasA calibration curve in ImHApf. (a) FTIR spectra comparison among different HasA concentrations and (b) calibration curve line close to validation line, revealing HasA concentrations in ImHApf within the range of $1750-1450 \mathrm{~cm}^{-1}$ : the two sets of data represent suitability for the immobilization to $16-18 \mathrm{mg}$ HasA.

\subsection{Catalytic Efficiency of ImHApf}

\subsubsection{Continuous Reuse of ImHApf}

ImHApf $(10 \mathrm{mg})$ was applied with substrate concentrations/reaction times of $0.8 \mathrm{mM} / 50 \mathrm{~h}$, $1.2 \mathrm{mM} / 60 \mathrm{~h}$, and $1.6 \mathrm{mM} / 70 \mathrm{~h}$ in a $50 \mathrm{mM}$ glycine-NaOH buffer $(4.0 \mathrm{~mL})$. After $50 \mathrm{~h}$ reactions, ketone-1 and (S)-1 in mixture were extracted with hexane $(4 \mathrm{~mL})$, and additional rac-1 was added to the mixture and allowed to incubate for an additional $50 \mathrm{~h} \times 3$. The results indicated that the activity was inversely proportional to the substrate concentration $(0.8 \mathrm{mM} / 50 \mathrm{~h}>1.2 \mathrm{mM} / 60 \mathrm{~h}>1.6 \mathrm{mM} / 70 \mathrm{~h})$, suggesting that the condition of $0.8 \mathrm{mM} / 50 \mathrm{~h}$ permitted reuse for three rounds of hexane extraction with stability and convenience in continuous operation. In addition, it was confirmed that, in the condition of ImHApf after multiple washing or reaction cycles using FTIR analysis, the HasA concentration was gradually reduced, suggesting that HasA was slightly leached from ImHApf (Figure 4). As shown in Figure 4, the isomer $(S)-\mathbf{1}(0.8 \mathrm{mM}$; $99 \%$ enantiomeric excess (ee), approximately $50 \%$ yield) was obtained from rac-1 $(1.6 \mathrm{mM})$ using ImHApf $(10 \mathrm{mg})$, leaving the highly enantiopure $R-(-)-\mathbf{1}(>99 \%$ ee: approximately $50 \%$ chemical yield).

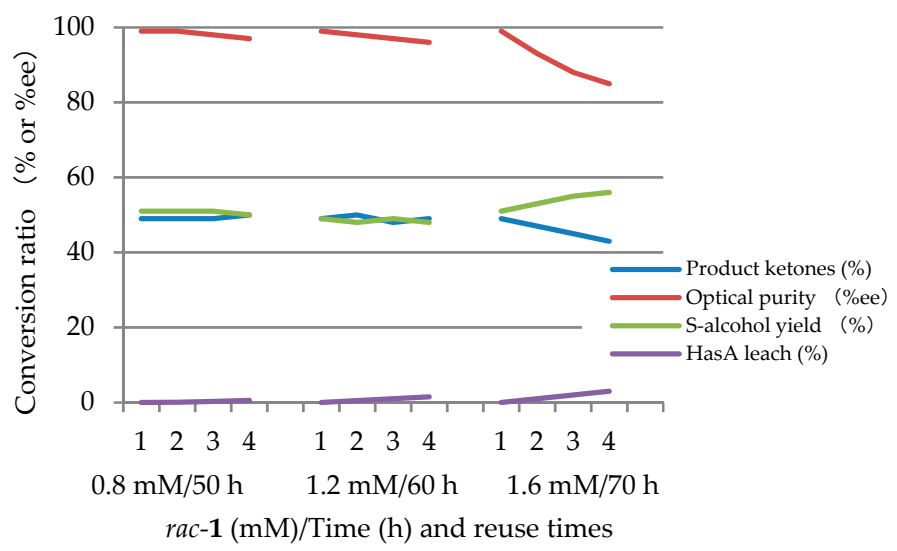

Figure 4. Number of reuses (1-4) of ImHApf (10 mg) under different substrate concentration/reaction time conditions $(0.8 \mathrm{mM} / 50 \mathrm{~h}, 1.2 \mathrm{mM} / 60 \mathrm{~h}$, and $1.6 \mathrm{mM} / 70 \mathrm{~h})$, revealing that ImHApf can be continuously reused even if treated with an organic solvent. 


\subsubsection{Unit of Activity Regarding ImHApf}

To investigate the activity regarding efficiency or turnover number, ImHApf was produced by suspending Toyonites $(1.0 \mathrm{~g})$ in a $50 \mathrm{mM}$ buffer $(\mathrm{pH}$ 9) containing HasApf $(18 \mathrm{mg} / 10 \mathrm{~mL})$ for $50 \mathrm{~h}$. The asymmetric oxidation activity of $\operatorname{ImHApf}(0.5,1,3$, and $6 \mathrm{mg}) / \mathrm{rac}-1$ (1.2 $\mathrm{mM}$ or $1.6 \mathrm{mM})$ in a $50 \mathrm{mM}$ glycine- $\mathrm{NaOH}$ buffer $(\mathrm{pH} 9.0 ; 4.0 \mathrm{~mL})$ at $40{ }^{\circ} \mathrm{C}$ was examined under magnetic stirring (700 rpm, 40-60 h). Figure 5 (left side) shows the dependence of $\Delta$ ketone (mM) per time (h), which is the ketone concentration $(\mathrm{mM})$ of the product on the reaction time (h) for each ImHApf quantity. In addition, Figure 5 (right side) shows the dependence of instant velocity $(\mathrm{mM} / \mathrm{h}$, referring to the maximum rate) on the reaction time (h) on each ImHApf quantity $(\mathrm{mg})$. The data indicate the catalytic efficiency of HasA; thus, these results indicate that the activity of ImHApf is approximately $0.74 \pm 0.03 \mathrm{mU} /(\mathrm{mg} \cdot \mathrm{min})$, and the turnover number was determined to be approximately $2 \times 10^{6}$. Thus, $0.5 \mathrm{mg}$ of ImHApf containing approximately $0.0004 \mu \mathrm{M}$ of enzyme catalyzes the formation of $0.8 \mathrm{mM}$ ketone, placing the maximum observed number of turnovers per active site at $2 \times 10^{6}$, as shown in Figure $5 b$. Therefore, HasApf immobilization utilizing Toyonite $(0.74 \mathrm{mU})$ was beneficial to enzyme efficiency in a comparison to the previous results in a HasApf-complex eluted from pea protein $(0.6 \mathrm{mU})[1]$.

(a) $0.53 \mathrm{mU}(\mathrm{mmol} /(4 \mathrm{~mL} \cdot \mathrm{min}))$ in the scale of $1.2 \mathrm{mM} / 4 \mathrm{~mL}$

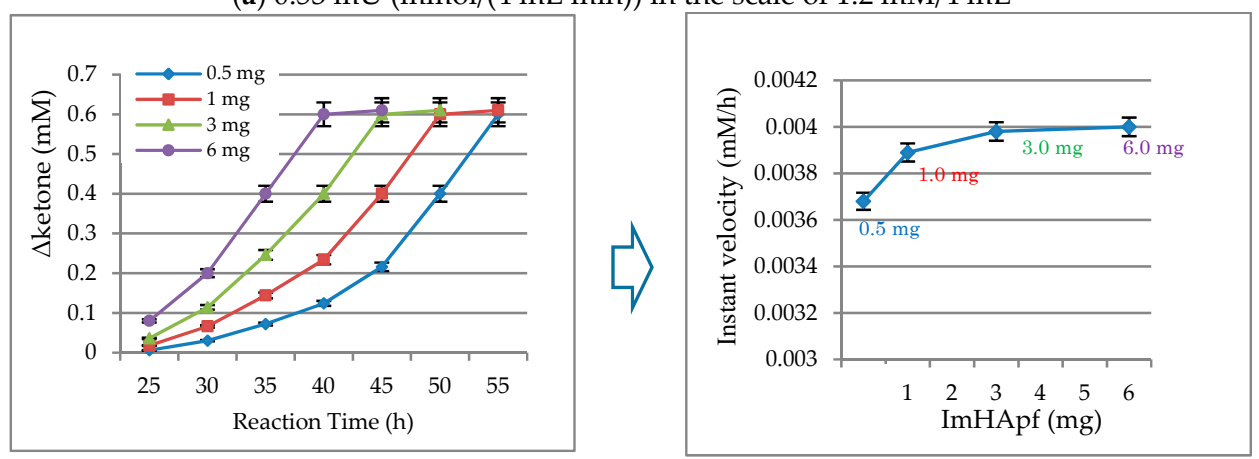

(b) $0.74 \mathrm{mU}(\mathrm{mmol} /(4 \mathrm{~mL} \cdot \mathrm{min}))$ in the scale of $1.6 \mathrm{mM} / 4 \mathrm{~mL}$
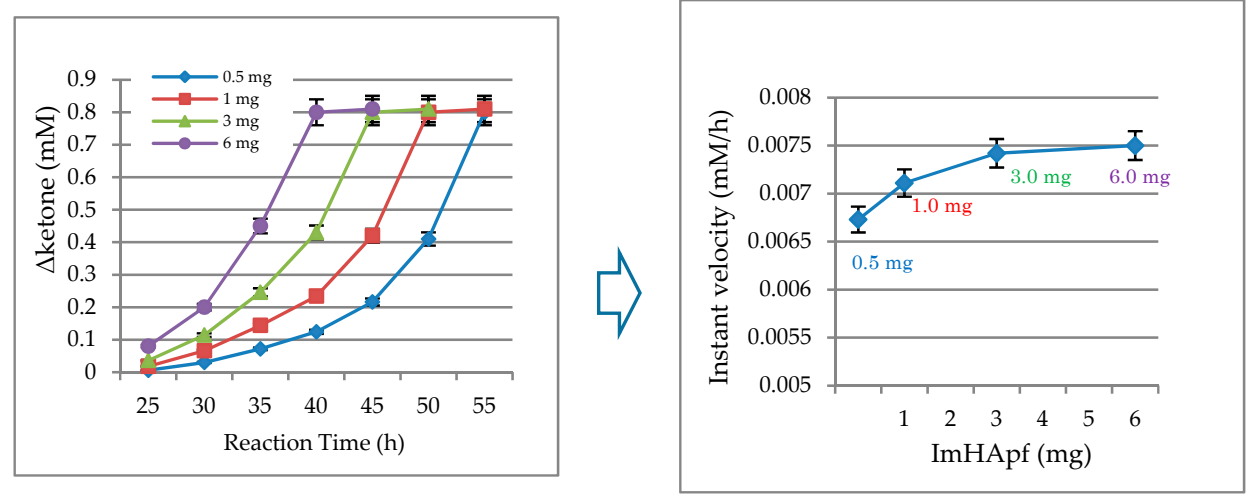

Figure 5. Comparison of the instant velocity $(\mathrm{mM} / \mathrm{h})$ per time $(\mathrm{h})$ in the different concentrations of (a) $1.2 \mathrm{mM}$ and (b) $1.6 \mathrm{mM}$ between rac-1 and ImHApf quantities $(0.5,1,3$, and $6 \mathrm{mg})$, i.e., the activity is $0.74 \pm 0.03 \mathrm{mU} /(\mathrm{mg} \cdot \mathrm{min})$, and the turnover number is approximately $2 \times 10^{6}$. Error bars represent mean \pm standard deviation (SD) $(n=4)$.

\subsection{Kinetic Resolution}

\subsubsection{Substrate-Specific Reactivities to the $m$ - and $p$-Substituted Groups}

The substrate-specific reactivities of each enantiomer of $r a c-\mathbf{1}$, rac-2, and $m$ - and $p$-substituted rac-1-phenyl ethanols (rac-3ab-6ab: $1.6 \mathrm{mM})$ on the oxidative activity of $\operatorname{ImHApf}(2 \mathrm{mg})$ were examined. After preincubation $(1 \mathrm{~h})$ of ImHApf with a $50 \mathrm{mM}$ glycine- $\mathrm{NaOH}$ buffer $(4.0 \mathrm{~mL})$ at $40{ }^{\circ} \mathrm{C}$ on 
a magnetic stirrer at $700 \mathrm{rpm}$ for 40-60 h, the solution reacted with racemic-1 and $\mathbf{- 2}$ and the other racemates (3ab-6ab). (R)-1 and -2 in racemic-1 and -2 oxidize highly enantioselectively by ImHApf; (S)-isomer was obtained with an enantiomer excess over $99 \%$ and a chemical yield of almost $50 \%$. As shown in Table 1, it was confirmed that the $(R)$-isomers in $m$ - and $p$-substituted rac-1-phenyl ethanols ( $r a c-3 a b-6 a b: 1.6 \mathrm{mM}$ ) using ImHApf also oxidize enantioselectively to corresponding ketones; therefore, the other (S)-3ab-6ab could be obtained with high enantioselectivities. These results newly showed that the substrate-specific reactivity using ImHApf could be achieved not only in naphthyl groups ( $r a c-1$ and -2) but also through the reaction of the racemate with $m$ - and $p$-substituted aryl methyl carbinol (rac-3ab-6ab).

Table 1. Asymmetric resolution of $\mathrm{rac}-\mathrm{ArCH}(\mathrm{OH}) \mathrm{Me}(\mathrm{Ar}=2-\mathrm{Np}$ and 6-MeO-2-Np, 3-Ar, and 4-Ar) with HasApf/ImHApf $(2 \mathrm{mg}, 0.0016 \mu \mathrm{M})$ under a substrate concentration $(1.6 \mathrm{mM})$.

\begin{tabular}{|c|c|c|c|c|c|c|c|}
\hline \multicolumn{3}{|c|}{ Substrate } & \multirow{2}{*}{$\begin{array}{l}\text { Reaction } \\
\text { Time (h) }\end{array}$} & \multirow{2}{*}{ Origin } & \multicolumn{3}{|c|}{ Product } \\
\hline Racemic-ArCH(OH)R & Ar & $\mathbf{R}$ & & & Compound & OP $(\% \text { ee })^{a}$ & CY $(\%)^{b}$ \\
\hline 1 & 6-MeO-2-Np & $\mathrm{Me}$ & 50 & $\operatorname{ImHApf} \mathrm{c}$ & $(S)-\mathbf{1}$ & $>99$ & 50 \\
\hline 2 & $2-\mathrm{Np}$ & $\mathrm{Me}$ & 55 & ImHApf & $(S)-2$ & $>99$ & 50 \\
\hline $3 a$ & 3-Cl-Ar & $\mathrm{Me}$ & 50 & ImHApf & $(S)-\mathbf{3 a}$ & $>99$ & 50 \\
\hline $4 a$ & 3-Br-Ar & $\mathrm{Me}$ & 55 & ImHApf & $(S)-\mathbf{4 a}$ & $>99$ & 49 \\
\hline $5 a$ & 3-Me-Ar & $\mathrm{Me}$ & 60 & ImHApf & $(S)-\mathbf{5 a}$ & $>99$ & 50 \\
\hline $6 a$ & 3-MeO-Ar & $\mathrm{Me}$ & 50 & ImHApf & $(S)-6 \mathbf{a}$ & $>99$ & 50 \\
\hline $3 b$ & 4-Cl-Ar & $\mathrm{Me}$ & 50 & ImHApf & $(S)-\mathbf{3 b}$ & $>99$ & 50 \\
\hline $4 b$ & 4-Br-Ar & $\mathrm{Me}$ & 55 & ImHApf & $(S)-\mathbf{4 b}$ & $>99$ & 50 \\
\hline $5 b$ & 4-Me-Ar & $\mathrm{Me}$ & 60 & ImHApf & $(S)-5 \mathbf{b}$ & $>99$ & 49 \\
\hline $6 b$ & 4-MeO-Ar & $\mathrm{Me}$ & 50 & ImHApf & $(S)-\mathbf{6 b}$ & $>99$ & 50 \\
\hline
\end{tabular}

${ }^{a}$ Optical purity (OP) determined by gas chromatograph. ee: enantiomer excess; ${ }^{b}$ Chemical yield (CY);

c Immobilized HasA from Pseudomonas fluorescens.

\subsubsection{ImHApf-Cyclic Deracemization Using $\mathrm{NaBH}_{4}(50 \mathrm{mg} \times 3)$}

After ImHApf $(100 \mathrm{mg})$ was preincubated in a $50 \mathrm{mM}$ glycine-NaOH buffer $(300 \mathrm{~mL})$ on a rotary shaker $(55 \mathrm{rpm})$ at $40{ }^{\circ} \mathrm{C}$ for $1 \mathrm{~h}$, the substrate ( $\mathrm{rac}-\mathbf{1}$ or -2: $45 \mathrm{mg}$ ) was mixed with the reaction solution and was further incubated for $40 \mathrm{~h}$; then, an additional substrate (55 mg) was added and it was incubated for an additional $20 \mathrm{~h}$. First results are reported in Table 2 in the substrate specificity of ImHApf, that is, only the $(R)$-isomer can be stereoselectively oxidized to the corresponding ketone, yielding a $50 \%$ chemical yield of $(S)$-isomer at $>99 \%$ ee. Then, ImHApf-deracemization using $\mathrm{NaBH}_{4}(50 \mathrm{mg} \times 3)$ was performed in the one-pot incubations conducted in $10 \mathrm{~h}$ intervals in Table 2 (second, third and fourth). Table 2 shows that ImHApf (100 mg) can only oxidize the $(R)$-isomer to the correspondent ketone (50\% chemical yield), which can be deracemized by $\mathrm{NaBH}_{4}$, thereby enabling obtainment in a single enantiomer $((S)-\mathbf{1}$ or -2 : $>99 \%$ ee) and $>90 \%$ ketone yield $(<4 \%)$.

Table 2. Application of ImHApf to cyclic deracemization using $\mathrm{NaBH}_{4}(50 \mathrm{mg} \times 3)$.

\begin{tabular}{|c|c|c|c|c|c|c|c|c|}
\hline & \multicolumn{8}{|c|}{ Deracemization: ImHApf $(100 \mathrm{mg}) / \mathrm{rac}-1$ (100 mg)/50 mM glycine-NaOH Buffer $(300 \mathrm{~mL})$} \\
\hline & $\mathrm{NaBH}_{4}$ & Time (h) & $\%$ Ketone & $\%$ Alcohol $^{\text {a }}$ & $\% R$-isomer & $\%$ S-isomer & Compound & $\%$ ee \\
\hline \multirow{2}{*}{ 1st } & \multirow{2}{*}{0} & \multirow{2}{*}{60} & \multirow{2}{*}{50} & \multirow{2}{*}{50} & 1.6 & 98.4 & $(S)-1^{\mathrm{b}}$ & 96.8 \\
\hline & & & & & 1.2 & 98.8 & $(S)-2^{c}$ & 97.6 \\
\hline \multirow{2}{*}{ 2nd } & \multirow{2}{*}{$50 \mathrm{mg}$} & \multirow{2}{*}{80} & \multirow{2}{*}{32} & \multirow{2}{*}{68} & 1.1 & 98.9 & (S)-1 & 97.8 \\
\hline & & & & & 0.9 & 99.1 & $(S)-2$ & 98.2 \\
\hline \multirow{2}{*}{$3 \mathrm{rd}$} & \multirow{2}{*}{$50 \mathrm{mg}$} & \multirow{2}{*}{90} & \multirow{2}{*}{17} & \multirow{2}{*}{83} & 0.7 & 99.3 & $(S)-1$ & 98.6 \\
\hline & & & & & 0.5 & 99.5 & $(S)-2$ & 99.0 \\
\hline \multirow{2}{*}{ 4th } & \multirow{2}{*}{$50 \mathrm{mg}$} & \multirow{2}{*}{100} & \multirow{2}{*}{4} & \multirow{2}{*}{96} & 0.4 & 99.6 & $(S)-1$ & 99.2 \\
\hline & & & & & 0.2 & 99.8 & $(S)-2$ & 99.6 \\
\hline
\end{tabular}

ImHApf-deracemization can be achieved by $\mathrm{NaBH}_{4}$ addition. ${ }^{a}$ Chemical yield (\%); ${ }^{\mathrm{b}}$ 6-MeO-2-Np; ${ }^{\mathrm{c}}$ 2-Np. 


\section{Discussion}

ImHApf deracemization using $\mathrm{NaBH}_{4}$ was successful, and it is suggested herein that the HasA immobilization into porous ceramic particles may help to employ a deracemization process in conjunction with a chemical reducing agent for the production of biochemicals such as pharmaceuticals, agricultural chemicals, and their precursors [6]. The explorations of efficiency and turnover number are also needed for the application of ImHApf to organic synthesis [26]. In enzyme kinetics, it is interesting to clarify the maximum number of substrate molecules that can be converted into product per catalytic site for a given concentration of enzyme per unit time [27], although $\mathrm{K}_{\mathrm{m}} / \mathrm{V}_{\max }$ value is not applicable because of the lower substrate concentrations $(<2 \mathrm{mM})$ and longer reaction times. The results in the present work newly indicate (1) the construction of a FTIR calibration curve using different concentrations of HasA in ImHApf; (2) an evaluation of the turnover number (approximately $2 \times 10^{6}$ ) and activity $[0.74 \pm 0.03 \mathrm{mU} /(\mathrm{mg} \cdot \mathrm{min})]$; and (3) clarification of the cyclic deracemization process using ImHApf and $\mathrm{NaBH}_{4}$.

Therefore, the novelty of ImHApf deracemization featuring continuous reusability has recently been proposed for heterogeneous enzyme catalysis [28], in which a redox cofactor (i.e., heme) is immobilized to perform asymmetric oxidation with the advantages of stability, reusability, convenience in continuous operation, and volumetric productivity. In the future, the ability of HasA in ImHApf to enantioselectively oxidize (synthesizing optically active sec-alcohols) is expected to become an important tool for building an environmentally friendly system that promotes industrial sustainability.

\section{Materials and Methods}

\subsection{Catalyst Preparation}

\subsubsection{ImHApf Preparation with HasApf Protein Expression/Purification}

HasApf was expressed in E. coli BL21(DE3) cells transformed with plasmid pET28a encoding bacterial genomic DNA (P. fluorescens) as previously reported [21]. For the preparation of immobilized HasA/ImHApf (approximately $0.0016 \mu \mathrm{M} / \mathrm{mg}$ ), Toyonites (i.e., 200, 200A, 200P, or 200M: Toyo Denka Kogyo Co., Ltd., Kochi, Japan) (1.0 g) were suspended in a HasApf/potassium phosphate solution (18 mg/10 mL) for $50 \mathrm{~h}$ at room temperature, utilizing a Recipro shaker NR-10 (Taitec Co., Ltd., Saitama, Japan) with the speed set to $111 \mathrm{~min}^{-1}$. ImHApf was recovered by filtration, dried under vacuum $\left[-50{ }^{\circ} \mathrm{C} / 10 \mathrm{~Pa}(1 \mathrm{~h}) \rightarrow 5^{\circ} \mathrm{C} / \mathrm{min} \rightarrow+50{ }^{\circ} \mathrm{C} / 10 \mathrm{~Pa}(3 \mathrm{~h})\right]$ utilizing an RLE-II 203 instrument (Nissei Limited Co., Ltd., Tokyo, Japan), and stored in a desiccator. The filtrate was assessed using FTIR analysis for HasA leach (\%), indicating the degree of HasA (\%) that remains after filtrating the suspension.

\subsubsection{General Scale of the Reactions Using HasApf and ImHApf}

The reaction mixture of ImHApf $(0.5 \mathrm{mg}-6.0 \mathrm{mg}) / \mathrm{rac}-\mathbf{1}, \mathbf{- 2}$, or -3ab-6ab $(0.8 \mathrm{mg}-1.6 \mathrm{mM})$ containing a $50 \mathrm{mM}$ glycine- $\mathrm{NaOH}$ buffer $(4.0 \mathrm{~mL})$ in an $18 \mathrm{~mm} \times 15 \mathrm{~mL}$ test tube was reacted at $40{ }^{\circ} \mathrm{C}$ under magnetic stirring at $700 \mathrm{rpm}$, i.e., the substrate solution $(10 \mu \mathrm{L}, 0.8 \mathrm{mM})$ that was prepared using rac-1, -2, or rac-3ab-6ab (1000 mg) in 2-propanol as a cosolvent (2.5 mL, 40,000 ppm) was dissolved, and the reactions were performed using HasA/ImHApf, approximately $0.0016 \mu \mathrm{M} / \mathrm{mg} / \mathrm{substrate}$ ( rac-1, -2, or -3ab-6ab (1.6 mM:0.8 mg)), in a $50 \mathrm{mM}$ glycine-NaOH buffer $(4.0 \mathrm{~mL})$ for $50 \mathrm{~h}$ at suitable reaction conditions (namely, with a turnover number of approximately $2 \times 10^{6}$ ). The reaction mixture containing a $50 \mathrm{mM}$ glycine-NaOH buffer $(300 \mathrm{~mL})$ in an Erlenmeyer flask $(500 \mathrm{~mL})$ was reacted at $40{ }^{\circ} \mathrm{C}$ under magnetic stirring at $500 \mathrm{rpm}$ by mixing the substrate solution $(0.25 \mathrm{~mL}, 40,000 \mathrm{ppm})$ and ImHApf (100 mg). 


\subsubsection{Enantiomeric Excess of Isolated Sec-Alcohols}

The calculations of enantiomeric excess, isolated from the reaction of $r a c-1, r a c-2$, or $r a c-m$ - and p-substituted rac-1-phenyl ethanols ( $r a c-3 a b-6 a b: 0.8 \mathrm{mM}$ or $1.2 \mathrm{mM}$ ) were performed for rac-1 using a Daicel Chiralcel OB-H column (Tokyo, Japan), for rac-2 using a Daicel Chiralpak AS-H column connected to a high performance liquid chromatography (HPLC) LC-10A system (Shimadzu Co., Ltd., Kyoto, Japan), and for rac-3ab-6ab using a gas chromatography with a Chrompack Chirasil-Dex CB capillary column (25 m $0.25 \mathrm{~mm}$ TC-5HT-fused silica-coated Agilent J \& W CP Chirasil-Dex CB: Tokyo, Japan). The analytical conditions of $r a c-\mathbf{1}, \mathbf{- 2}$, or $r a c-3 \mathbf{a b}-\mathbf{6 a b}$, including mobile phase, flow rate, and temperature, have been previously reported in detail $[9,21]$.

\subsubsection{FTIR Analyses Using ImHApf}

A portable type of ATR instrument was applied for FTIR analysis (A2 Technologies, ML version). After ImHApf samples were placed on the diamond crystal of ATR, FTIR spectra could be obtained via a pressure device (a single bounce reflection method), and the calibration curve of HasApf in ImHApf could then be constructed in the range of $1750-1450 \mathrm{~cm}^{-1}$ using Panorama software [15].

\subsubsection{Specific Activity and Turnover Efficiency on ImHApf}

The activity of ImHApf was calculated using different concentrations of HasApf immobilized with Toyonite $200(1 \mathrm{~g})$ for each HasApf/potassium phosphate solution $(18 \mathrm{mg} / 10 \mathrm{~mL})$. The different volumes of ImHApf $(0.5,1,3$, and $6 \mathrm{mg})$ were reacted with rac-1 $(1.6 \mathrm{mM})$ in a $50 \mathrm{mM}$ glycine- $\mathrm{NaOH}$ buffer (4.0 mL; pH 9.0) via magnetic stirring (700 rpm) for $0.8 \mathrm{mM} / 50 \mathrm{~h}, 1.2 \mathrm{mM} / 60 \mathrm{~h}$, or $1.6 \mathrm{mM} / 70 \mathrm{~h}$. This value indicates the quantity of enzyme $(\mathrm{mg})$ that is capable of oxidizing $1 \mu \mathrm{mol}$ of $\mathrm{rac}-\mathbf{1}$ per minute. The formulae of instant velocity $(\mathrm{mM} / \mathrm{h})$, activity $[\mathrm{mmol} /(4 \mathrm{~mL} \cdot \mathrm{min})]$, or specific activity \{unit $[\mathrm{mmol} /(4 \mathrm{~mL} \cdot \mathrm{min})]\}$ have been previously reported [1].

\section{Conclusions}

ImHApf has continuous reusability in the asymmetric oxidation activity even if treated with an organic solvent, and its catalytic efficiencies are evaluated using the turnover number (approximately $2 \times 10^{6}$ ) and activity $[0.74 \pm 0.03 \mathrm{mU} /(\mathrm{mg} \cdot \mathrm{min})]$, suggesting that iron in ImHApf is oxidized by dissolved oxygen, leading to the promotion of oxoferryl $(\mathrm{Fe}(\mathrm{IV})=\mathrm{O})$ species. The kinetic resolution of $(S)-(+)-\mathbf{1}$ or $\mathbf{- 2}$ or $\mathbf{- 3} \mathbf{a b}-\mathbf{6 a b}$ ( $>99 \%$ ee, approximately $50 \%$ chemical yield) from $\mathrm{rac}-\mathbf{1}$ or $\mathbf{- 2}$ or $\mathbf{- 3} \mathbf{a b}-\mathbf{6} \mathbf{a b}$ catalyzes via the selective oxidation of $(R)-(-)-\mathbf{1}$ or $\mathbf{- 2}$ or $\mathbf{- 3} \mathbf{a b}-\mathbf{6} \mathbf{a b}$ to the corresponding ketone. The ketone produced from $(R)-(-)-\mathbf{1}$ or $\mathbf{- 2}(100 \mathrm{mg})$ was deracemized by $\mathrm{NaBH}_{4}(50 \mathrm{mg} \times 3)$ using ImHApf (100 mg) and obtained as a pure enantiomer ( $>99 \%$ ee, approximately $90 \%$ chemical yield) in the absence of an added cofactor, e.g., $\mathrm{NAD}(\mathrm{P})$, in the aqueous medium at $40{ }^{\circ} \mathrm{C}$. Therefore, HasAs with an immobilized porous ceramic particle are expected to become important asymmetric oxidation catalysts in organic synthesis, building environmentally friendly systems that promote industrial sustainability.

Acknowledgments: The author would like to thank the General Manager Koh Ueda from Wako Pure Chemical Industries Ltd. for placing SanCat-Super (namely, ImHApf) on the market (Code No. 350-42331 (250 mg)). This study was fully funded by Sanyo Shokuhin Co., Ltd., Gunma, Japan.

Conflicts of Interest: The author declares no conflict of interest.

\section{References}

1. Nagaoka, H. Application of a heme-binding protein eluted from encapsulated biomaterials to the catalysis of enantioselective oxidation. ACS Catal. 2014, 4, 553-565. [CrossRef]

2. Ozaki, S.; Nakahara, A.; Sato, T. Mechanism of heme uptake by heme acquisition system A. Chem. Lett. 2011, 40, 362-363. [CrossRef] 
3. Ozaki, S.; Sato, A.; Migita, T.C.; Uchida, T.; Ishimori, K. Spectroscopic studies on HasA from Yersinia pseudotuberculosis. J. Inorg. Biochem. 2014, 138, 31-38. [CrossRef] [PubMed]

4. Krieg, S.; Huché, F.; Diederichs, K.; Izadi-Pruneyre, N.; Lecroisey, A.; Wandersman, P.C. Heme uptake across the outer membrane as revealed by crystal structures of the receptor-hemophore complex. PNAS 2009, 106, 1045-1050. [CrossRef] [PubMed]

5. Kamori, M.; Hori, T.; Yamashita, Y.; Hirose, Y.; Naoshima, Y. Immobilization of lipase on a new inorganic ceramics support, toyonite, and the reactivity and enantioselectivity of the immobilized lipase. J. Mol. Catal. B Enzym. 2000, 9, 269-274. [CrossRef]

6. Magnan, E.; Catarino, I.; Paolucci-Jeanjean, D.; Belleville, M.P. Immobilization of lipase on a ceramic membrane: Activity and stability. J. Membr. Sci. 2004, 241, 161-167. [CrossRef]

7. Shoji, O.; Fujishiro, T.; Nakajima, H.; Kim, M.; Nagano, S.; Shiro, Y.; Watanabe, Y. Hydrogen peroxide dependent monooxygenations by tricking the substrate recognition of cytochrome P450 $\mathrm{BS} \beta$. Angew. Chem. Int. Ed. 2007, 46, 3656-3659. [CrossRef] [PubMed]

8. Nagaoka, H. Chiral resolution function with immobilized food proteins. Biotechnol. Prog. 2003, 19, 1149-1155. [CrossRef] [PubMed]

9. Nagaoka, H. Ability of different biomaterials to enantioselectively catalyze oxidation and reduction reactions. Biotechnol. Prog. 2004, 20, 128-133. [CrossRef] [PubMed]

10. Nagaoka, H.; Udagawa, K.; Kirimura, K. Cross-linked protein complex exhibiting asymmetric oxidation activities in the absence of added cofactor. Biotechnol. Prog. 2012, 28, 953-961. [CrossRef] [PubMed]

11. Nagaoka, H. The application of a cytochrome P450 enzyme eluted from encapsulated for the catalysis of enantioselective oxidation. RSC Adv. 2014, 4, 16333-16344. [CrossRef]

12. Thakur, V.V.; Sudalai, A. Enantioselective synthesis of (S)- $\alpha$-arylpropionic acids via Pd-catalyzed kinetic resolution of benzylic alcohols. Indian J. Chem. Sect. B Org. Chem. Incl. Med. Chem. 2005, 44B, 557-561. [CrossRef]

13. Gillam, E.M.; Hayes, M.A. The evolution of cytochrome P450 enzymes as biocatalysts in drug discovery and development. Curr. Top. Med. Chem. 2013, 13, 2254-2280. [CrossRef] [PubMed]

14. Paulsen, I.T.; Press, C.M.; Ravel, J.; Kobayashi, D.Y.; Myers, G.S.; Mavrodi, D.V.; DeBoy, R.T.; Seshadri, R.; Ren, Q.; Madupu, R.; et al. Complete genome sequence of the plant commensal Pseudomonas fluorescens Pf-5. Nat. Biotechnol. 2005, 23, 873-878. [CrossRef] [PubMed]

15. Nagaoka, H. An HASApf-Redoxin Complex causing asymmetric catalytic oxidation via the regenerative formation of a reactive oxygen species. Dalton Trans. 2015, 44, 13384-13393. [CrossRef] [PubMed]

16. Du, J.F.; Li, W.; Li, L.; Wen, G.B.; Lin, Y.W.; Tan, X. Regulation the coordination state of a heme protein by a designed distal hydrogen-bonding network. ChemistryOpen 2015, 4, 97-101. [CrossRef] [PubMed]

17. Ortiz de Montellano, P.R. Cytochrome P450: Structure, Mechanism, and Biochemistry, 3rd ed.; Kluwer Academic/Plenum: New York, NY, USA, 2004.

18. Machii, K.; Watanabe, Y. Acylperoxo-Iron (III) porphyrin complexes: A new entry of potent oxidants for the alkene epoxidation. J. Am. Chem. Soc. 1995, 117, 6691-6697. [CrossRef]

19. Jepkorir, G.; Rodríguez, J.C.; Rui, H.; Im, W.; Lovell, S.; Battaile, K.P.; Alontaga, A.Y.; Yukl, E.T.; Moënne-Loccoz, P.; Rivera, M. Structural, NMR spectroscopic, and computational investigation of hemin loading in the hemophore HasAp from Pseudomonas aeruginosa. Am. Chem. Soc. 2010, 132, 9857-9872. [CrossRef] [PubMed]

20. Giles, M.N.; Watts, B.A.; Giles, M.N.; Watts, B.A.; Giles, I.G.; Fry, H.F.; Littlechild, A.J.; Jacob, C. Metal and redox modulation of cysteine protein function. Chem. Biol. 2003, 10, 677-693. [CrossRef]

21. Nagaoka, H. Heterogeneous asymmetric oxidation catalysis using hemophore HasApf. Application in the chemoenzymatic deracemization of sec-alcohols with sodium borohydride. Catalysts 2016, 6, 38. [CrossRef]

22. Steinreiber, J.; Faber, K.; Griengl, H. De-racemization of enantiomers versus de-epimerization of diastereomers-classification of dynamic kinetic asymmetric transformations (DYKAT). Chem. Eur. J. 2008, 14, 8060-8072. [CrossRef] [PubMed]

23. Turner, N.J. Deracemisation methods. Curr. Opin. Chem. Biol. 2010, 14, 115-121. [CrossRef] [PubMed]

24. Zhou, Y.; Bowler, B.E.; Lynch, K.; Eaton, S.S.; Eaton, G.R. Interspin distances in spin-labeled metmyoglobin variants determine by saturation recovery EPR. Biophys. J. 2000, 79, 1039-1052. [CrossRef]

25. Gruber, C.C.; Lavandera, I.; Faber, K.; Kroutil, W. From a Racemate to a Single Enantiomer: Deracemization by Stereoinversion. Adv. Synth. Catal. 2006, 348, 1789-1805. [CrossRef] 
26. Auld, D.S.; Bergman, T. The role of zinc for alcohol dehydrogenase structure and function. Cell Mol. Life Sci. 2008, 65, 3961-3970. [CrossRef] [PubMed]

27. Lam, M.K.; Lee, K.T.; Mohamed, A.R. Homogeneous, heterogeneous and enzymatic catalysis for transesterification of high free fatty acid oil (waste cooking oil) to biodiesel: A review. Biotechnol. Adv. 2010, 28, 500-518. [CrossRef] [PubMed]

28. Zhao, Z.Y.; Liu, J.; Hahn, M.; Qiao, S.; Middelberg, A.P.J.; He, L. Encapsulation of lipase in mesoporous silica yolk-shell spheres with enhanced enzyme stability. RSC Adv. 2013, 3, 22008-22013. [CrossRef]

(c) 2016 by the author; licensee MDPI, Basel, Switzerland. This article is an open access article distributed under the terms and conditions of the Creative Commons Attribution (CC-BY) license (http:/ / creativecommons.org/licenses/by/4.0/). 